MATHEMATICS OF COMPUTATION

Volume 66, Number 218, April 1997, Pages 527-545

S 0025-5718(97)00817-X

\title{
ON CONVERGENCE OF NUMERICAL SCHEMES FOR HYPERBOLIC CONSERVATION LAWS WITH STIFF SOURCE TERMS
}

\author{
ABDALLAH CHALABI
}

\begin{abstract}
We deal in this study with the convergence of a class of numerical schemes for scalar conservation laws including stiff source terms. We suppose that the source term is dissipative but it is not necessarily a Lipschitzian function. The convergence of the approximate solution towards the entropy solution is established for first and second order accurate MUSCL and for splitting semi-implicit methods.
\end{abstract}

\section{INTRODUCTION}

Many physical problems are governed by hyperbolic conservation laws with nonvanishing stiff source terms. These problems could describe the effect of relaxation as in the kinetic theory of gases, elasticity with memory, water waves, traffic flows... etc.

These problems can be mathematically described in the scalar case by the following Cauchy problem:

$$
\begin{gathered}
\left.u_{t}+f(u)_{x}=q(u), \quad(x, t) \in \mathbb{R} \times\right] 0, T[; T>0, \\
u(x, 0)=u_{0}(x), \quad x \in \mathbb{R} .
\end{gathered}
$$

In the case of relaxation phenomena, a theoretical study of these problems may be found in Chen et al. [4], Liu [13], Whitham [26].

Numerical methods have been derived for the approximation of the conservation laws including non-stiff source terms in [1], [3], [16], [19], [22]. These methods are based on explicit difference schemes. It is well known that explicit schemes are not appropriate for the numerical treatment of the stiff source terms, this motivates the use of semi-implicit and fully implicit schemes.

The approximation of the stiff case was recently studied by several authors (see $[2],[5],[7],[9],[10],[12],[16],[18],[19])$, where different methods like asymptotic or splitting methods are used.

The main difficulty, when we deal with numerical solution of stiff problems is the wrong location of the discontinuities. This problem has been investigated in [2], [5], [7], [9], [10], [12].

Received by the editor September 19, 1995 and, in revised form, March 29, 1996.

1991 Mathematics Subject Classification. Primary 35L65, 65M05, 65M10.

Key words and phrases. Conservation laws, stiff source term, Runge-Kutta method, splitting method, implicit scheme, TVD, TVB scheme, entropy solution. 
Error bounds related to the approximation of (1.1)-(1.2) were derived in [18], [19] and [22].

In this paper, we give a study of the convergence of the approximate solution obtained by semi-implicit, implicit and high-order semi-implicit schemes where the hyperbolic part is approximated using a monotone scheme. We also study the convergence of a second-order accurate semi-implicit splitting scheme proposed in Jin [9].

In this study, we do not assume that the source term $q$ is a Lipschitzian function with respect to $u$ but we suppose that $q^{\prime} \leq 0$. This hypothesis is realistic since it does mean the dissipativity of the source term $q$ in the sense of Chen et al. [4], which is the case in the models of combustion ([2], [7], [14]), gas dynamics with heat transfer [9] and water waves with friction force of the bottom [26] ... etc. We point out that in all these examples and many others, the source term is not Lipschitzian. The dissipativity of the source term is also assumed by Chen et al. in [4] and Liu in [13].

Thanks to the implicit character and to the dissipativity of the source term $q$ (nonpositivity of $q^{\prime}$ ), all our proposed schemes are TVD or TVB, entropy satisfying at the limit and they are monotone in the first-order accurate semi-implicit case. Then they almost possess all the properties as in the homogeneous schemes $(q=0)$.

This paper is organized as follows: the next section is devoted to recall some preliminaries related to the hyperbolic conservation laws including source terms. At first we give a result which summarizes the properties of the exact solution of the Cauchy problem when $q^{\prime}$ is nonpositive. These properties are similar to those related to the homogeneous case. In section 3, we give an analysis of a first order accurate semi-implicit scheme. Section 4 concerns the study of a fully implicit scheme. Section 5 is devoted to the study of a second-order accurate semi-implicit scheme. In section 6 , we present a second-order semi-implicit splitting method together with the study of the convergence of the approximate solution towards the entropy satisfying solution of the Cauchy problem (1.1)-(1.2). Finally in the last section, we give an extension of the semi-implicit schemes to the two-dimensional case.

\section{Preliminaries}

We seek a weak solution to the Cauchy problem (1.1)-(1.2), that is a function $u \in L^{\infty}(\mathbb{R} \times] 0, T[)$ satisfying:

$$
\begin{array}{r}
\int_{\mathbb{R}} \int_{] 0, T[}\left[u \varphi_{t}+f(u) \varphi_{x}\right] d x d t+\int_{\mathbb{R}} u_{0}(x) \varphi(x, 0) d x \\
=-\int_{\mathbb{R}} \int_{] 0, T[} q(u) \varphi(x, t) d x d t
\end{array}
$$

for all $\varphi \in \mathcal{C}^{1}(\mathbb{R} \times[0, T[)$, with compact support in $(\mathbb{R} \times[0, T[)$.

Let $\eta \in \mathcal{C}^{2}(\mathbb{R})$ be a strictly convex function, whose entropy flux function is $F$, that is

$$
\eta^{\prime}(u) f^{\prime}(u)=F^{\prime}(u), \quad \forall u \in \mathbb{R}
$$


The solution of (1.1)-(1.2) is not necessarily unique and the physical one is characterized by the following entropy condition:

$$
\int_{\mathbb{R}} \int_{] 0, T[}\left[\eta(u) \varphi_{t}+F(u) \varphi_{x}\right] d x d t \geq-\int_{\mathbb{R}} \int_{] 0, T[} \eta^{\prime}(u) q(u) \varphi(x, t) d x d t
$$

for all positive test functions $\varphi \in \mathcal{C}^{1}(\mathbb{R} \times] 0, T[)$, with compact support in $\left.\mathbb{R} \times\right] 0, T$.

Usually the solution $u$ lies in BV, where BV denotes the subspace of $L_{l o c}^{1}$ consisting of functions with bounded total variation

$$
T V(u)=\sup _{h \neq 0} \int_{\mathbb{R}} \frac{|u(x+h)-u(x)|}{|h|} d x .
$$

We observe that in the nonhomogeneous conservation laws case, the characteristic curves are not straight lines along which the solution $u$ is not necessarily constant.

Let $u(x, t)=S(t) u_{0}$ denote the unique weak solution of (1.1)-(1.2) which satisfies the entropy condition. Let us assume that

$$
\sup _{u} q^{\prime}(u) \leq \gamma
$$

We recall the following result:

Proposition 2.1 (see [11]). If $u_{0} \in L^{\infty}(\mathbb{R}) \cap L^{1}(\mathbb{R}), f \in \mathcal{C}^{1}(\mathbb{R})$, then the problem (1.1)-(1.2) possesses a unique entropy solution $u(x, t)=S(t) u_{0}$ satisfying

i) $\left\|S(t) u_{0}\right\|_{L^{\infty}(\mathbb{R})} \leq e^{\gamma t}\left(\left\|u_{0}\right\|_{L^{\infty}(\mathbb{R})}+|q(0)| t\right)$.

ii) Moreover, for any $v_{0} \in L^{\infty}(\mathbb{R})$ we have

$$
\left\|S(t) u_{0}-S(t) v_{0}\right\|_{L^{1}(\mathbb{R})} \leq e^{\gamma t}\left\|u_{0}-v_{0}\right\|_{L^{1}(\mathbb{R})} .
$$

iii) If $u_{0}(x) \leq v_{0}(x)$, then the corresponding solutions satisfy

$$
S(t) u_{0} \leq S(t) v_{0} .
$$

Using Proposition 2.1, we can prove the following:

Proposition 2.2. If $u_{0} \in L^{\infty}(\mathbb{R}) \cap L^{1}(\mathbb{R}), f \in \mathcal{C}^{1}(\mathbb{R}), q \in \mathcal{C}^{1}(\mathbb{R})$ such that $q(0)=$ 0 and $q^{\prime} \leq 0$, then the problem (1.1)-(1.2) possesses a unique entropy solution $u(x, t)=S(t) u_{0}$ satisfying

i) $\left\|S(t) u_{0}\right\|_{L^{\infty}(\mathbb{R})} \leq\left\|u_{0}\right\|_{L^{\infty}(\mathbb{R})}$.

ii) Moreover, for any $v_{0} \in L^{\infty}(\mathbb{R})$ we have

$$
\left\|S(t) u_{0}-S(t) v_{0}\right\|_{L^{1}(\mathbb{R})} \leq\left\|u_{0}-v_{0}\right\|_{L^{1}(\mathbb{R})} .
$$

iii) $T V\left(S(t) u_{0}\right) \leq T V\left(u_{0}\right)$.

iv) If $u_{0}(x) \leq v_{0}(x)$, then the corresponding solutions satisfy

$$
S(t) u_{0} \leq S(t) v_{0} .
$$

Proof. Taking $\gamma=0$ and $q(0)=0$ in Proposition 2.1, we easily obtain the inequalities i) and ii). We set $v_{0}(x)=u_{0}(x+h)$ in ii) of Proposition 2.1, divide by $|h|$ the inequality ii) in Proposition 2.1 and take the sup over $h$, to get inequality iii).

Property iv) is proved under the general hypothesis in Proposition 2.1. 
Remark 2.1. If we suppose that $q \in \mathcal{C}^{1}(\mathbb{R})$ such that $q(0)=0$ and $q^{\prime} \leq 0$, then $q$ is dissipative in the sense of Chen et al. [4], indeed if we take $\eta(u)=u^{2} / 2$ as an entropy function and apply the mean value theorem to $q$, we get

$$
\eta^{\prime}(u) q(u)=u\left[q(0)+u q^{\prime}(\xi)\right] ; \quad \min (0, u)<\xi<\max (0, u)=q^{\prime}(\xi) u^{2} \leq 0 .
$$

Remark 2.2. We observe that under the hypothesis of Proposition 2.2, the solution of (1.1)-(1.2) possesses the same properties as in the homogeneous case $(q=0)$.

Let $h$ be the spatial mesh size and $k$ be the time grid size related to $h$ by the fixed positive number $r$ through:

$$
r=k / h .
$$

A weak solution of (1.1)-(1.2) is approximated by a function $u_{h}$ defined on $\mathbb{R} \times] 0, T[$ by:

$$
u_{h}(x, t)=u_{j}^{n} \text { for }(x, t) \in I_{j} \times J_{n}
$$

with

$$
\begin{aligned}
\left.I_{j} \times J_{n}=\right](j-1 / 2) h,(j+1 / 2) h[\times] & (n-1 / 2) k,(n+1 / 2) k[ \\
\forall j & \in \mathbb{Z} \text { and } n \leq N=E(T / k)+1
\end{aligned}
$$

where $E$ denotes the integer part function.

With the notation $\Delta_{+} c_{j}=c_{j+1}-c_{j}$, we have:

$$
T V\left(u^{n}\right)=\sum_{j \in \mathbb{Z}}\left|\Delta_{+} u^{n}\right|
$$

and

$$
\left\|u^{n}\right\|_{L^{1}(\mathbb{Z})}=h \sum_{j \in \mathbb{Z}}\left|u_{j}^{n}\right| .
$$

A numerical scheme is TVD if the approximate solution satisfies:

$$
T V\left(u^{n+1}\right) \leq T V\left(u^{n}\right)
$$

and TVB if there exists a positive constant $C$ such that

$$
T V\left(u^{n+1}\right) \leq C
$$

Initial condition (1.2) is projected onto the space of piecewise constant functions as:

$$
u_{j}^{0}=1 / h \int_{I_{j}} u_{0}(x) d x \quad \forall j \in \mathbb{Z} .
$$

In all of the following, we assume that

$$
q \in \mathcal{C}^{1}(\mathbb{R}) \text { such that } q(0)=0 \text { and } q^{\prime} \leq 0 .
$$

Let $g=g(u, v)$ be a locally Lipschitz numerical flux of a three point conservative scheme, locally Lipschitz in both arguments and satisfying:

1) $g(u, v)$ is nonincreasing in $u$,

2) $g(u, v)$ is nondecreasing in $v$,

3) $g(u, u)=f(u)$.

The associated explicit scheme is monotone under the following CFL condition:

$$
r \max _{w, z}\{|g(u, w)-g(v, w)|+|g(z, u)-g(z, v)|\} \leq|u-v| \forall u, v \in \mathcal{A},
$$


where

$$
\mathcal{A}=\left\{v \in L^{\infty}(\mathbb{R}),\|v\|_{L^{\infty}(\mathbb{R})} \leq\left\|u_{0}\right\|_{L^{\infty}(\mathbb{R})}\right\} .
$$

Remark 2.3. The hypothesis on $g$ can be weakned by taking $g$ as a flux of an Escheme introduced by Osher in [15]. The cases where $g$ is the numerical flux of Godunov, Lax-Friedrichs, Engquist-Osher schemes are included in this study.

\section{SEmi-IMPlicit SCHEME}

In this section, we consider the approximation of (1.1)-(1.2), where we handle the source term using implicit schemes and keep explicit the approximation of the hyperbolic part.

For the approximation of problem (1.1)-(1.2), we consider the semi-implicit scheme:

$$
u_{j}^{n+1}=u_{j}^{n}-r\left[g\left(u_{j}^{n}, u_{j+1}^{n}\right)-g\left(u_{j-1}^{n}, u_{j}^{n}\right)\right]+\Delta t q\left(u_{j}^{n+1}\right) .
$$

Let us set

$$
\bar{u}_{j}^{n+1}=u_{j}^{n}-r\left[g\left(u_{j}^{n}, u_{j+1}^{n}\right)-g\left(u_{j-1}^{n}, u_{j}^{n}\right)\right] .
$$

Then the scheme (3.1) may be written

$$
u_{j}^{n+1}=\bar{u}_{j}^{n+1}+\Delta t q\left(u_{j}^{n+1}\right) .
$$

For the properties of this scheme, we prove:

Proposition 3.1. If the CFL condition (2.8) is satisfied, then

i) The scheme (3.1) is monotone.

ii) $\left\|u^{n+1}\right\|_{L^{\infty}(\mathbb{Z})} \leq\left\|u^{n}\right\|_{L^{\infty}(\mathbb{Z})}$.

iii) $T V\left(u^{n+1}\right) \leq T V\left(u^{n}\right)$.

Proof. To show that the scheme (3.1) is monotone, let us suppose that

$$
u_{j}^{n} \leq v_{j}^{n}, \forall j \in \mathbb{Z}
$$

Using (3.3), we have

$$
u_{j}^{n+1}-v_{j}^{n+1}=\bar{u}_{j}^{n+1}-\bar{v}_{j}^{n+1}+\Delta t q\left(u_{j}^{n+1}\right)-\Delta t q\left(v_{j}^{n+1}\right) .
$$

Hence

$$
\left[u_{j}^{n+1}-v_{j}^{n+1}\right]\left[1-\Delta t q^{\prime}\left(\alpha_{j}^{n}\right)\right]=\bar{u}_{j}^{n+1}-\bar{v}_{j}^{n+1} .
$$

Since the scheme (3.2) is monotone under the CFL condition (2.8), then

$$
u^{n} \leq v^{n} \Rightarrow \bar{u}^{n+1} \leq \bar{v}^{n+1} .
$$

Using the nonpositivity of $q^{\prime}$, we obtain

$$
u_{j}^{n+1} \leq v_{j}^{n+1} \forall j \in \mathbb{Z} .
$$

Taking into account the CFL condition (2.8), we show easily that

$$
\left\|\bar{u}^{n+1}\right\|_{L^{\infty}(\mathbb{Z})} \leq\left\|u^{n}\right\|_{L^{\infty}(\mathbb{Z})}
$$

and

Using (3.3), we have

$$
T V\left(\bar{u}^{n+1}\right) \leq T V\left(u^{n}\right)
$$

Thus

$$
u_{j}^{n+1}-\Delta t q\left(u_{j}^{n+1}\right)=\bar{u}_{j}^{n+1}
$$

$$
\left(1-q^{\prime}\left(\xi_{j}^{n}\right)\right) u_{j}^{n+1}=\bar{u}_{j}^{n+1}
$$


But $q^{\prime}$ is non-positive, then we obtain:

$$
\left\|u^{n+1}\right\|_{L^{\infty}(\mathbb{Z})} \leq\left\|\bar{u}^{n+1}\right\|_{L^{\infty}(\mathbb{Z})}
$$

and

$$
T V\left(u^{n+1}\right) \leq T V\left(\bar{u}^{n+1}\right) .
$$

Using the former inequalities, we get

$$
\left\|u^{n+1}\right\|_{L^{\infty}(\mathbb{Z})} \leq\left\|u^{n}\right\|_{L^{\infty}(\mathbb{Z})}
$$

and

$$
T V\left(u^{n+1}\right) \leq T V\left(u^{n}\right)
$$

Let us now return to the existence of a solution of (3.1).

Proposition 3.2. If $u^{0} \in L^{\infty}(\mathbb{Z})$, then the scheme (3.1) admits a unique solution $\left(u^{n+1}\right) \in L^{\infty}(\mathbb{Z})$.

Proof. The function

$$
x \longmapsto x-\Delta t q(x)
$$

is differentiable and strictly monotone since $q^{\prime} \leq 0$.

Then the equation

$$
u_{j}^{n+1}-\Delta t q\left(u_{j}^{n+1}\right)=\bar{u}_{j}^{n+1}
$$

admits a unique solution $u_{j}^{n+1}$.

By Proposition 3.1, this solution $u^{n+1}$ belongs to $L^{\infty}(\mathbb{Z})$ if $u^{0}$ belongs to $L^{\infty}(\mathbb{Z})$.

Remark 3.1. One can show easily that if $u_{0}$ belongs to $L^{\infty}(\mathbb{R})$, then $u^{0}$ belongs to $L^{\infty}(\mathbb{Z})$.

Theorem 3.1. If $u_{0} \in L^{\infty}(\mathbb{R}) \cap L^{1}(\mathbb{R}), f \in \mathcal{C}^{1}(\mathbb{R}), q \in \mathcal{C}^{1}(\mathbb{R})$ such that $q(0)=0$ and $q^{\prime} \leq 0$, then under the CFL condition (2.8) the approximate solution $u_{h}$ constructed by the semi-implicit scheme (3.1) converges in $L_{\text {loc }}^{1}(\mathbb{R} \times] 0, T[)$ towards the entropy satisfying solution of (1.1)-(1.2), as $h$ tends to zero.

Proof. From Proposition 3.1, the sequence $\left(u_{h}\right)$ is bounded in $L^{\infty}(\mathbb{R} \times] 0, T[) \cap$ $B V(\mathbb{R} \times] 0, T[)$, then by Helly's theorem, we can extract a subsequence still labelled $\left(u_{h}\right)$ which converges towards $u$ in $L_{l o c}^{1}(\mathbb{R} \times] 0, T[)$. Let us now prove that $u$ satisfies the entropy condition (2.3). To do this we write the semi-implicit scheme (3.1) in the form:

$$
\begin{gathered}
\bar{u}_{j}^{n+1}=u_{j}^{n}-r\left[g\left(u_{j}^{n}, u_{j+1}^{n}\right)-g\left(u_{j-1}^{n}, u_{j}^{n}\right)\right], \\
u_{j}^{n+1}=\bar{u}_{j}^{n+1}+\Delta t q\left(u_{j}^{n+1}\right) .
\end{gathered}
$$

The scheme (3.2) is monotone (under the CFL condition (2.8)), then using a result of Tadmor [21], there exists a numerical entropy flux $F_{j-1 / 2}$ associated with the entropy $\eta$ such that

$$
\eta\left(\bar{u}_{j}^{n+1}\right)-\eta\left(u_{j}^{n}\right)+r \Delta_{+} F_{j-1 / 2}^{n} \leq 0 .
$$

From (3.7) and the convexity of $\eta$ we obtain

$$
\eta\left(u_{j}^{n+1}\right)-\eta\left(u_{j}^{n}\right)+r \Delta_{+} F_{j-1 / 2}^{n} \leq \eta^{\prime}\left(u_{j}^{n+1}\right)\left(u_{j}^{n+1}-\bar{u}_{j}^{n+1}\right) .
$$


Multipying (3.8) by $\varphi_{j}^{n} h\left(\varphi_{j}^{n}=\varphi(j h, n k)\right)$ ( $\varphi$ being any positive test function with compact support) and taking the sum over $j$ and $n$, we get

$$
\begin{aligned}
\sum_{n, j} h\left(\eta\left(u_{j}^{n+1}\right)-\eta\left(u_{j}^{n}\right)\right) & \varphi_{j}^{n}+\sum_{n, j} k \Delta_{+}\left(F_{j-1 / 2}^{n}\right) \varphi_{j}^{n} . \\
& \leq \sum_{n, j} h \eta^{\prime}\left(u_{j}^{n+1}\right)\left(u_{j}^{n+1}-\bar{u}_{j}^{n+1}\right) \varphi_{j}^{n} .
\end{aligned}
$$

We denote by $A$ (resp. $B$ ) the left-hand side (resp. the right-hand side) of the inequality (3.9). Using discrete integration by parts we get

$$
A=-\sum_{n, j} k h\left(\eta\left(u_{j}^{n+1}\right)\left(\varphi_{j}^{n+1}-\varphi_{j}^{n}\right) / k-\sum_{n, j} h k F_{j+1 / 2}^{n}\left(\varphi_{j+1}^{n}-\varphi_{j}^{n}\right) / h .\right.
$$

Hence

$$
A=-\int_{\mathbb{R}} \int_{] 0 . T[}\left[\eta\left(u_{h}(x, t)\right)\left(\varphi_{h}(x, t)\right)_{t}+F_{h}(x, t)\left(\varphi_{h}(x, t)\right)_{x}\right] d x d t
$$

where

$$
\begin{aligned}
\left.F_{h}(x, t)=F_{j-1 / 2}^{n} \text { if }(x, t) \in\right] & (j-1) h, j h[\times] n k,(n+1) k[ \\
& \forall j \in \mathbb{Z} \text { and } n \leq N=E(T / k)+1 .
\end{aligned}
$$

Making use of Lebesgue's theorem we prove that

$$
\begin{gathered}
\int_{\mathbb{R}} \int_{] 0 . T[}\left[\eta\left(u_{h}(x, t)\right)\left(\varphi_{h}(x, t)\right)_{t}+F_{h}(x, t) \varphi(x, t)_{x}\right] d x d t \\
\longrightarrow \int_{\mathbb{R}} \int_{] 0 . T[}\left[\eta(u) \varphi_{t}+F(u) \varphi_{x}\right] d x d t
\end{gathered}
$$

Let us now return to the limit of the right-hand side $B$ of (3.9)

$$
\begin{aligned}
B= & \left.\sum_{n, j} h \eta^{\prime}\left(u_{j}^{n+1}\right)\left(u_{j}^{n+1}-\bar{u}_{j}^{n+1}\right)\right) \varphi_{j}^{n} \\
& =\sum_{n, j} k h \eta^{\prime}\left(u_{j}^{n+1}\right) q\left(u_{j}^{n+1}\right) \varphi_{j}^{n} .
\end{aligned}
$$

Hence

$$
B=\int_{\mathbb{R}} \int_{] 0, T[} \eta^{\prime}\left(u_{h}\right) q\left(u_{h}\right) \varphi_{h} d x d t
$$

as $h$ tends to 0

$$
B \longrightarrow \int_{\mathbb{R}} \int_{] 0 . T[} \eta^{\prime}(u) q(u) \varphi d x d t
$$

Then the limit $u$ (of the subsequence) is a weak solution which satisfies the entropy condition (2.3). The entropy solution being unique, the whole sequence $\left(u_{h}\right)$ converges towards the entropy solution of (1.1)-(1.2) as the step $h$ tends to zero.

Remark 3.2. Under other hypotheses on $q^{\prime}$ and $f^{\prime}$, a similar result to the former one, was proved by Schroll et al. in [19].

Remark 3.3. For the numerical solution of scheme (3.1), one can use a Newton method to linearise the source term, that is:

$$
q\left(u_{j}^{n+1}\right)=q\left(u_{j}^{n}\right)+q^{\prime}\left(u_{j}^{n}\right)\left(u_{j}^{n+1}-u_{j}^{n}\right) .
$$




\section{Fully IMPLiCIT SCHEME}

Now, let us consider a fully implicit scheme in the form:

$$
u_{j}^{n+1}=u_{j}^{n}-r\left[g\left(u_{j}^{n+1}, u_{j+1}^{n+1}\right)-g\left(u_{j-1}^{n+1}, u_{j}^{n+1}\right)\right]+\Delta t q\left(u_{j}^{n+1}\right) .
$$

First, we introduce as in [25], the operator $T_{\nu}$ defined on piecewise constant functions by

$$
\left(T_{\nu}\left(u_{h}\right)\right)_{j}=u_{j}-\nu r\left[g\left(u_{j}, u_{j+1}\right)-g\left(u_{j-1}, u_{j}\right)\right], \forall j \in \mathbb{Z},
$$

where $\nu$ is a parameter satisfying $\nu>0$, and $u_{h}=\left(u_{j}\right)_{j \in \mathbb{Z}}$.

The following result is proved in [25].

Lemma $4.1([25])$. Let $u_{h}=\left(u_{j}\right)_{j \in \mathbb{Z}}$ be a piecewise constant function. If the positive parameter $\nu$ is sufficiently small such that

$$
\nu r \max _{w, z}|g(u, w)-g(v, w)|+|g(z, u)-g(z, v)| \leq|u-v| \forall u, v \in \mathcal{A},
$$

then

i) $\left\|T_{\nu}\left(u_{h}\right)\right\|_{L^{\infty}(\mathbb{Z})} \leq\left\|u_{h}\right\|_{L^{\infty}(\mathbb{Z})} \cdot$

ii) $T V\left(T_{\nu}\left(u_{h}\right)\right) \leq T V\left(u_{h}\right)$.

Next, we prove

Proposition 4.1. The approximate solution given by (4.1) satisfies the following properties:

i) $\left\|u^{n+1}\right\|_{L^{\infty}(\mathbb{Z})} \leq\left\|u^{n}\right\|_{L^{\infty}(\mathbb{Z})}$,

ii) $T V\left(u^{n+1}\right) \leq T V\left(u^{n}\right)$.

Proof. i) Using the operator $T_{\nu}$, the scheme (4.1) may be written:

$$
u^{n+1}=\frac{\nu}{1+\nu} u^{n}+\frac{1}{1+\nu} T_{\nu}\left(u^{n+1}\right)+\frac{\nu}{1+\nu} \Delta t q\left(u^{n+1}\right) .
$$

Applying the mean value theorem to $q$, we get

$$
\left[1-\frac{\nu}{1+\nu} \Delta t q^{\prime}\left(\xi^{n+1}\right)\right] u^{n+1}=\frac{\nu}{1+\nu} u^{n}+\frac{1}{1+\nu} T_{\nu}\left(u^{n+1}\right) .
$$

Taking into account the non-positivity of $q^{\prime}$, we obtain

$$
\left\|u^{n+1}\right\|_{L^{\infty}(\mathbb{Z})} \leq \frac{\nu}{1+\nu}\left\|u^{n}\right\|_{L^{\infty}(\mathbb{Z})}+\frac{1}{1+\nu}\left\|T_{\nu}\left(u^{n+1}\right)\right\|_{L^{\infty}(\mathbb{Z})} .
$$

By making use of Lemma 4.1, we have

$$
\left\|T_{\nu}\left(u^{n+1}\right)\right\|_{L^{\infty}(\mathbb{Z})} \leq\left\|u^{n+1}\right\|_{L^{\infty}(\mathbb{Z})} .
$$

Hence

$$
\left\|u^{n+1}\right\|_{L^{\infty}(\mathbb{Z})} \leq \frac{\nu}{1+\nu}\left\|u^{n}\right\|_{L^{\infty}(\mathbb{Z})}+\frac{1}{1+\nu}\left\|u^{n+1}\right\|_{L^{\infty}(\mathbb{Z})}
$$

Thus

$$
\frac{\nu}{1+\nu}\left\|u^{n+1}\right\|_{L^{\infty}(\mathbb{Z})} \leq \frac{\nu}{1+\nu}\left\|u^{n}\right\|_{L^{\infty}(\mathbb{Z})} .
$$

Then

$$
\left\|u^{n+1}\right\|_{L^{\infty}(\mathbb{Z})} \leq\left\|u^{n}\right\|_{L^{\infty}(\mathbb{Z})} .
$$


ii) From (4.1), we have

$$
\begin{aligned}
u_{j+1}^{n+1}-u_{j}^{n+1}= & \frac{\nu}{1+\nu}\left(u_{j+1}^{n}-u_{j}^{n}\right)+\frac{1}{1+\nu}\left[\left(T_{\nu}\left(u^{n+1}\right)\right)_{j+1}-\left(T_{\nu}\left(u^{n+1}\right)\right)_{j}\right] \\
& +\frac{\nu}{1+\nu} \Delta t\left[q\left(u_{j+1}^{n+1}\right)-q\left(u_{j}^{n+1}\right)\right] .
\end{aligned}
$$

Hence

$$
\begin{aligned}
& {\left[1-\frac{\nu}{1+\nu} \Delta t q^{\prime}\left(\xi_{j+1 / 2}^{n+1}\right)\right]\left(u_{j+1}^{n+1}-u_{j}^{n+1}\right)} \\
& \quad=\frac{\nu}{1+\nu}\left(u_{j+1}^{n}-u_{j}^{n}\right)+\frac{1}{1+\nu}\left[\left(T_{\nu}\left(u^{n+1}\right)\right)_{j+1}-\left(T_{\nu}\left(u^{n+1}\right)\right)_{j}\right] .
\end{aligned}
$$

$q^{\prime}$ being non-positive, we get

$$
\left|u_{j+1}^{n+1}-u_{j}^{n+1}\right| \leq \frac{\nu}{1+\nu}\left|u_{j+1}^{n}-u_{j}^{n}\right|+\frac{1}{1+\nu}\left|\left[\left(T_{\nu}\left(u^{n+1}\right)\right)_{j+1}-\left(T_{\nu}\left(u^{n+1}\right)\right)_{j}\right]\right| .
$$

Summing up the last inequality over $j \in \mathbb{Z}$, we obtain

$$
T V\left(u^{n+1}\right) \leq \frac{\nu}{1+\nu} T V\left(u^{n}\right)+\frac{1}{1+\nu} T V\left(T_{\nu}\left(u^{n+1}\right)\right) .
$$

Using Lemma 4.1 again, we obtain

Hence

$$
T V\left(u^{n+1}\right) \leq \frac{\nu}{1+\nu} T V\left(u^{n}\right)+\frac{1}{1+\nu} T V\left(u^{n+1}\right) .
$$

Then

$$
\frac{\nu}{1+\nu} T V\left(u^{n+1}\right) \leq \frac{\nu}{1+\nu} T V\left(u^{n}\right)
$$

$$
T V\left(u^{n+1}\right) \leq T V\left(u^{n}\right) .
$$

That is, the scheme (4.1) is TVD.

Proposition 4.2. The scheme (4.1) admits a unique solution $u^{n+1} \in L^{\infty}(\mathbb{Z})$.

Proof. The scheme (4.1) may be written in the form

$$
u_{j}^{n+1}+r\left[g\left(u_{j}^{n+1}, u_{j+1}^{n+1}\right)-g\left(u_{j-1}^{n+1}, u_{j}^{n+1}\right)\right]-\Delta t q\left(u_{j}^{n+1}\right)=u_{j}^{n} .
$$

Let us introduce the operator $T: L^{\infty}(\mathbb{Z}) \longrightarrow L^{\infty}(\mathbb{Z})$ defined by

$$
T(u)_{j}=u_{j}+r\left[g\left(u_{j}, u_{j+1}\right)-g\left(u_{j-1}, u_{j}\right)\right]-\Delta t q\left(u_{j}\right) \forall j \in \mathbb{Z} .
$$

To prove the existence of a solution scheme (4.1), it suffices to prove that the operator $T$ is invertible. To do this, we show that there exists a positive constant $C$ such that

We have

$$
\left\|T^{\prime}(u) w\right\|_{L^{\infty}(\mathbb{Z})} \geq C\|w\|_{L^{\infty}(\mathbb{Z})} \forall w \in L^{\infty}(\mathbb{Z}) .
$$

$$
\begin{aligned}
{\left[T^{\prime}(u) w\right]_{j}=} & w_{j}+r g_{u}^{\prime}\left(u_{j}, u_{j+1}\right) w_{j}+r g_{v}^{\prime}\left(u_{j}, u_{j+1}\right) w_{j+1} \\
& -r g_{u}^{\prime}\left(u_{j-1}, u_{j}\right) w_{j-1}-r g_{v}^{\prime}\left(u_{j-1}, u_{j}\right) w_{j}-\Delta t q^{\prime}\left(u_{j}\right) w_{j}
\end{aligned}
$$

Let

$$
T^{\prime}(u) w=b
$$

with

$$
b_{j}=\left[1+a_{j+1 / 2}-b_{j-1 / 2}-\Delta t q^{\prime}\left(u_{j}\right)\right] w_{j}-a_{j-1 / 2} w_{j-1}+b_{j+1 / 2} w_{j+1} ;
$$

thus

$$
\left|b_{j}\right| \geq\left[1+a_{j+1 / 2}-b_{j-1 / 2}-\Delta t q^{\prime}\left(u_{j}\right)\right]\left|w_{j}\right|-a_{j-1 / 2}\left|w_{j-1}\right|+b_{j+1 / 2}\left|w_{j+1}\right| .
$$


Taking the sup over $j \in \mathbb{Z}$, in the last inequality, we get

$$
\|b\|_{L^{\infty}(\mathbb{Z})} \geq\|w\|_{L^{\infty}(\mathbb{Z})},
$$

that is

$$
\left\|T^{\prime}(u) w\right\|_{L^{\infty}(\mathbb{Z})} \geq\|w\|_{L^{\infty}(\mathbb{Z})}
$$

then there exists a unique solution of $(4.1)$ in $L^{\infty}(\mathbb{Z})$.

Theorem 4.1. If $u_{0} \in L^{\infty}(\mathbb{R}) \cap L^{1}(\mathbb{R}), f \in \mathcal{C}^{1}(\mathbb{R}), q \in \mathcal{C}^{1}(\mathbb{R})$ such that $q(0)=0$ and $q^{\prime} \leq 0$, then the approximate solution $u_{h}$ constructed by the implicit scheme (4.1), converges in $L_{\text {loc }}^{1}(\mathbb{R} \times] 0, T[)$ towards the entropy satisfying solution of (1.1)(1.2), as $h$ tends to zero.

Proof. Using Proposition (4.1), the family of the approximate solution $\left(u_{h}\right)$ is bounded in $L^{\infty}(\mathbb{R} \times] 0, T[) \cap B V(\mathbb{R} \times] 0, T[)$ then by Helly's theorem, we can extract a subsequence still labelled $\left(u_{h}\right)$ which converges towards $u$ in $L_{l o c}^{1}(\mathbb{R} \times] 0, T[)$. We show now that $u$ satisfies the entropy condition (2.3).

Let $\eta$ be a convex entropy function, with entropy flux $F(u)$. Multiplying (4.1) by $\eta^{\prime}\left(u_{j}^{n+1}\right)$, we obtain

$$
\begin{gathered}
\eta^{\prime}\left(u_{j}^{n+1}\right)\left(u_{j}^{n+1}-u_{j}^{n}\right)+r \eta^{\prime}\left(u_{j}^{n+1}\right)\left[g\left(u_{j}^{n+1}, u_{j+1}^{n+1}\right)-g\left(u_{j-1}^{n+1}, u_{j}^{n+1}\right)\right] \\
=\Delta t \eta^{\prime}\left(u_{j}^{n+1}\right) q\left(u_{j}^{n+1}\right)
\end{gathered}
$$

The function $\eta$ being convex, then

$$
\eta\left(u_{j}^{n+1}\right)-\eta\left(u_{j}^{n}\right) \leq \eta^{\prime}\left(u_{j}^{n+1}\right)\left(u_{j}^{n+1}-u_{j}^{n}\right) ;
$$

thus, (4.4) gives us

$$
\begin{aligned}
\eta\left(u_{j}^{n+1}\right)-\eta\left(u_{j}^{n}\right)+r \eta^{\prime}\left(u_{j}^{n+1}\right)\left[g \left(u_{j}^{n+1},\right.\right. & \left.\left.u_{j+1}^{n+1}\right)-g\left(u_{j-1}^{n+1}, u_{j}^{n+1}\right)\right] \\
& \leq \Delta t \eta^{\prime}\left(u_{j}^{n+1}\right) q\left(u_{j}^{n+1}\right) .
\end{aligned}
$$

Next, we add to both sides of (4.5) the quantity

$$
\Delta_{+} \tilde{F}\left(u_{j}\right)=\Delta_{+}\left(F\left(u_{j}\right)+\eta^{\prime}\left(u_{j}\right)\left[g\left(u_{j-1}, u_{j}\right)-f\left(u_{j}\right)\right]\right) .
$$

We now obtain

$$
\begin{aligned}
\eta\left(u_{j}^{n+1}\right)-\eta\left(u_{j}^{n}\right) & +r \Delta_{+} \tilde{F}\left(u_{j}\right) \leq-r\left(\Delta_{+} \eta^{\prime}\left(u_{j}^{n+1}\right)\right)\left[g\left(u_{j}^{n+1}, u_{j+1}^{n+1}\right)\right] . \\
& -\Delta_{+}\left[\eta^{\prime}\left(u_{j}^{n+1}\right) f\left(u_{j}\right)-F\left(u_{j}\right)\right]+\Delta t \eta^{\prime}\left(u_{j}^{n+1}\right) q\left(u_{j}^{n+1}\right) .
\end{aligned}
$$

We have

$$
\begin{aligned}
\Delta_{+} F\left(u_{j}\right) & =\int_{u_{j}^{n+1}}^{u_{j+1}^{n+1}} F^{\prime}(w) d w=\int_{u_{j}^{n+1}}^{u_{j+1}^{n+1}} \eta^{\prime}(w) f^{\prime}(w) d w \\
& =\Delta_{+}\left[\eta^{\prime}\left(u_{j}^{n+1}\right) f\left(u_{j}^{n+1}\right)\right]-\int_{u_{j}^{n+1}}^{u_{j+1}^{n+1}} \eta^{\prime \prime}(w) f(w) d w .
\end{aligned}
$$

and

$$
\left.\Delta_{+} \eta^{\prime}\left(u_{j}^{n+1}\right)\right)\left[g\left(u_{j}^{n+1}, u_{j+1}^{n+1}\right)\right]=\int_{u_{j}^{n+1}}^{u_{j+1}^{n+1}}\left[g\left(u_{j}^{n+1}, u_{j+1}^{n+1}\right)\right] \eta^{\prime \prime}(w) d w
$$


Substituting (4.7) and (4.8) into (4.6), we get

$$
\begin{gathered}
\eta\left(u_{j}^{n+1}\right)-\eta\left(u_{j}^{n}\right)+r \Delta_{+} \tilde{F}\left(u_{j}\right) \leq \int_{u_{j}^{n+1}}^{u_{j+1}^{n+1}} \eta^{\prime \prime}(w)\left[g\left(u_{j}^{n+1}, u_{j+1}^{n+1}\right)-f(w)\right] d w \\
+\Delta t \eta^{\prime}\left(u_{j}^{n+1}\right) q\left(u_{j}^{n+1}\right) .
\end{gathered}
$$

Thanks to the hypotheses on the signs of $g_{u}^{\prime}$ and $g_{v}^{\prime}$ the first term of the righthand side of (4.9) is nonpositive, then

$$
\eta\left(u_{j}^{n+1}\right)-\eta\left(u_{j}^{n}\right)+r \Delta_{+} \tilde{F}\left(u_{j}\right) \leq \Delta t \eta^{\prime}\left(u_{j}^{n+1}\right) q\left(u_{j}^{n+1}\right) .
$$

As in the proof of Theorem 3.1, multiplying (4.10) by $\varphi_{j}^{n} h\left(\varphi_{j}^{n}=\varphi(j h, n k)\right)$ and taking the sum over $j$ and $n$, we get at the limit (as $h$ tends to zero)

$$
\int_{\mathbb{R}} \int_{] 0, T[}\left[\eta(u) \varphi_{t}+F(u) \varphi_{x}\right] d x d t \geq-\int_{\mathbb{R}} \int_{] 0, T[} \eta^{\prime}(u) q(u) \varphi(x, t) d x d t
$$

that is the limit $u$ satisfies the entropy condition.

Remark 4.1. We point out that the former result was proved without any CFL condition.

\section{MUSCL TYPE SEMI-IMPLICIT SCHEME}

To construct quasi second-order accurate scheme, as in [23],[24], we assume that the initial values form a piecewise linear distribution:

$$
u^{n}(x)=u_{j}^{n}+\left(x-x_{j}\right) \delta_{j}^{n}, x_{j-1 / 2}<x<x_{j+1 / 2},
$$

where

$$
\delta_{j}^{n}=\left\{\begin{array}{c}
\sigma \operatorname{Min}\left\{\left|\hat{\delta}_{j}^{n}\right|,\left|u_{j+1}^{n}-u_{j}^{n}\right| / h,\left|u_{j}^{n}-u_{j-1}^{n}\right| / h\right\} \\
\quad \text { if } \sigma=\operatorname{sgn}\left(\hat{\delta}_{j}^{n}\right)=\operatorname{sgn}\left(u_{j+1}^{n}-u_{j}^{n}\right)=\operatorname{sgn}\left(u_{j}^{n}-u_{j-1}^{n}\right), \\
0 \quad \text { otherwise }
\end{array}\right.
$$

with

From (5.2) there exist $\alpha_{j+1 / 2}^{n}$ and $\beta_{j-1 / 2}^{n}$ such that

$$
\hat{\delta}_{j}^{n}=\left(u_{j+1}^{n}-u_{j-1}^{n}\right) /(2 h) .
$$

$$
0 \leq \alpha_{j+1 / 2}^{n} \leq 1 \quad \text { and } \quad 0 \leq \beta_{j-1 / 2}^{n} \leq 1
$$

and

$$
\delta_{j}^{n}=\alpha_{j+1 / 2}^{n}\left(u_{j+1}^{n}-u_{j}^{n}\right) / h=\beta_{j-1 / 2}^{n}\left(u_{j}^{n}-u_{j-1}^{n}\right) / h
$$

We will need the initial boundary values inside cell $j$ :

$$
\left\{\begin{array}{l}
u_{j+1 / 2,-}^{n}=u_{j}^{n}+h / 2 \delta_{j}^{n}, \\
u_{j-1 / 2,+}^{n}=u_{j}^{n}-h / 2 \delta_{j}^{n} .
\end{array}\right.
$$

The semi-implicit scheme may be written:

$$
u_{j}^{n}=u_{j}^{n}-r\left[g\left(u_{j+1 / 2,-}^{n}, u_{j+1 / 2,+}^{n}\right)-g\left(u_{j-1 / 2,-}^{n}, u_{j-1 / 2,+}^{n}\right)\right]+k q\left(u_{j}^{n+1}\right) .
$$


Let

$$
\mathcal{A}=\left\{v \in L^{\infty}(\mathbb{R}),\|v\|_{L^{\infty}(\mathbb{R})} \leq\left\|u_{0}\right\|_{L^{\infty}(\mathbb{R})}\right\} .
$$

Proposition 5.1. Under the CFL condition:

$$
r \max _{w, z}\{|g(u, w)-g(v, w)|+|g(z, u)-g(z, v)|\} \leq 2 / 3|u-v| \forall u, v \in \mathcal{A}
$$

the approximate solution given by the semi-implicit scheme (5.5) satisfies

$$
\begin{aligned}
& \text { i) }\left\|u^{n+1}\right\|_{L^{\infty}(\mathbb{Z})} \leq\left\|u^{n}\right\|_{L^{\infty}(\mathbb{Z})}, \\
& \text { ii) } T V\left(u^{n+1}\right) \leq T V\left(u^{n}\right) .
\end{aligned}
$$

Proof. The scheme (5.5) may be written in the form

$$
u_{j}^{n+1}=\hat{u}_{j}^{n+1}+k q\left(u_{j}^{n+1}\right)
$$

where

$$
\hat{u}_{j}^{n+1}=u_{j}^{n}-r\left[g\left(u_{j+1 / 2,-}^{n}, u_{j+1 / 2,+}^{n}\right)-g\left(u_{j-1 / 2,-}^{n}, u_{j-1 / 2,+}^{n}\right)\right] .
$$

Using (5.3), the incremental form of the scheme (5.8) gives

$$
\hat{u}_{j}^{n+1}=u_{j}^{n}-C_{j-1 / 2}^{n}\left(u_{j}^{n}-u_{j-1}^{n}\right)+D_{j+1 / 2}^{n}\left(u_{j+1}^{n}-u_{j}^{n}\right)
$$

where

$$
\left\{\begin{array}{l}
C_{j-1 / 2}^{n}=r a_{j,-}^{n}\left(1+1 / 2 \beta_{j-1 / 2}^{n}-1 / 2 \alpha_{j-1 / 2}^{n}\right), \\
D_{j+1 / 2}^{n}=-r b_{j,+}^{n}\left(1-1 / 2 \beta_{j+1 / 2}^{n}+1 / 2 \alpha_{j+1 / 2}^{n},\right.
\end{array}\right.
$$

where

$$
a_{j,-}^{n}=\left[g\left(u_{j+1 / 2,-}^{n}, u_{j+1 / 2,+}^{n}\right)-g\left(u_{j-1 / 2,-}^{n}, u_{j+1 / 2,+}^{n}\right)\right] /\left(u_{j+1 / 2,-}^{n}-u_{j-1 / 2,-}^{n}\right)
$$

and

$$
b_{j,+}^{n}=\left[g\left(u_{j-1 / 2,-}^{n}, u_{j+1 / 2,+}^{n}\right)-g\left(u_{j-1 / 2,-}^{n}, u_{j-1 / 2,+}^{n}\right)\right] /\left(u_{j+1 / 2,+}^{n}-u_{j-1 / 2,+}^{n}\right) .
$$

Under the CFL condition (5.6), we have

$$
D_{j+1 / 2}^{n} \geq 0, C_{j-1 / 2}^{n} \geq 0
$$

and

$$
D_{j+1 / 2}^{n}+C_{j+1 / 2}^{n} \leq 1
$$

Hence

$$
\left\|\hat{u}^{n+1}\right\|_{L^{\infty}(\mathbb{Z})} \leq\left\|u^{n}\right\|_{L^{\infty}(\mathbb{Z})}
$$

and

$$
T V\left(\hat{u}^{n+1}\right) \leq T V\left(u^{n}\right) .
$$

Taking account of $q^{\prime} \leq 0$ and using the same method as in the proof of Proposition 3.1, we get the properties i) and ii).

Proposition 5.2. If $u^{0} \in L^{\infty}(\mathbb{Z})$, then the scheme (5.5) admits a unique solution $\left(u^{n+1}\right) \in L^{\infty}(\mathbb{Z})$. 
The proof of Propositon 5.2 is similar to that of Proposition 3.2.

To prove the convergence of the approximate solution towards the entropy solution, we modify slightly the limitation formula (5.2) by taking:

$$
\delta_{j}^{n}=\left\{\begin{array}{c}
\sigma \operatorname{Min}\left\{\left|\hat{\delta}_{j}^{n}\right|,\left|u_{j+1}^{n}-u_{j}^{n}\right| / h,\left|u_{j}^{n}-u_{j-1}^{n}\right| / h, C h^{\alpha-1}\right\} \\
\text { if } \sigma=\operatorname{sgn}\left(\hat{\delta}_{j}^{n}\right)=\operatorname{sgn}\left(u_{j+1}^{n}-u_{j}^{n}\right)=\operatorname{sgn}\left(u_{j}^{n}-u_{j-1}^{n}\right), \\
\quad \text { otherwise, }
\end{array}\right.
$$

where $C>0 ; 1>\alpha>0$.

Next, we prove

Theorem 5.1. If $u_{0} \in L^{\infty}(\mathbb{R}) \cap L^{1}(\mathbb{R}), f \in \mathcal{C}^{1}(\mathbb{R}), q \in \mathcal{C}^{1}(\mathbb{R})$, such that $q(0)=$ 0 and $q^{\prime} \leq 0$, then under the CFL condition (5.6), the approximate solution $u_{h}$ constructed by the scheme (5.5) converges in $L_{\text {loc }}^{1}(\mathbb{R} \times] 0, T[)$ towards the entropy satisfying solution of (1.1)-(1.2), as $h$ tends to zero.

Proof. Making use of Proposition 5.1, we show that $\left(u_{h}\right)$ constructed by the scheme (5.5) is bounded in $L^{\infty}(\mathbb{R} \times] 0, T[) \cap B V(\mathbb{R} \times] 0, T[)$; then by Helly's theorem, like in the first-order accurate case, we can extract a subsequence still labelled $\left(u_{h}\right)$ which converges towards a weak solution $u$ of (1.1)-(1.2), in $L_{l o c}^{1}(\mathbb{R} \times] 0, T[)$. To prove that $u$ is the entropy solution, we rewrite the scheme (5.5) in the form:

$$
\hat{u}_{j}^{n+1}=u_{j}^{n}-r\left[g\left(u_{j}^{n}, u_{j+1}^{n}\right)-g\left(u_{j-1}^{n}, u_{j}^{n}\right)\right],
$$

$$
\begin{aligned}
u_{j}^{n+1}=\hat{u}_{j}^{n+1}-r[ & \left(g\left(u_{j+1 / 2,-}^{n}, u_{j+1 / 2,+}^{n}\right)-g\left(u_{j}^{n}, u_{j+1}^{n}\right)\right) \\
& \left.-\left(g\left(u_{j-1 / 2,-}^{n}, u_{j-1 / 2,+}^{n}\right)+g\left(u_{j-1}^{n}, u_{j}^{n}\right)\right)\right]+k q\left(u_{j}^{n+1}\right) .
\end{aligned}
$$

The scheme (5.12-a) is monotone under the CFL condition (5.6), then using a result of Tadmor [21], there exists a numerical entropy flux $F_{j-1 / 2}$ associated with the entropy $\eta$ such that

$$
\eta\left(\hat{u}_{j}^{n+1}\right)-\eta\left(u_{j}^{n}\right)+r \Delta_{+} F_{j-1 / 2}^{n} \leq 0
$$

From (5.13) and the convexity of $\eta$ we obtain

$$
\eta\left(u_{j}^{n+1}\right)-\eta\left(u_{j}^{n}\right)+r \Delta_{+} F_{j-1 / 2}^{n} \leq \eta^{\prime}\left(u_{j}^{n+1}\right)\left(u_{j}^{n+1}-\hat{u}_{j}^{n+1}\right) .
$$

Multipying (5.14) by $\varphi_{j}^{n} h\left(\varphi_{j}^{n}=\varphi(j h, n k)\right)$ and taking the sum over $j$ and $n$, we get

$$
\begin{aligned}
\sum_{n, j} h\left(\eta\left(u_{j}^{n+1}\right)-\eta\left(u_{j}^{n}\right)\right) & \varphi_{j}^{n}+\sum_{n, j} k \Delta_{+}\left(F_{j-1 / 2}^{n}\right) \varphi_{j}^{n} \\
\leq & \left.\sum_{n, j} h \eta^{\prime}\left(u_{j}^{n+1}\right)\left(u_{j}^{n+1}-\hat{u}_{j}^{n+1}\right)\right) \varphi_{j}^{n} ;
\end{aligned}
$$


thus

$$
\begin{aligned}
\sum_{n, j} h( & \left.\left(u_{j}^{n+1}\right)-\eta\left(u_{j}^{n}\right)\right) \varphi_{j}^{n}+\sum_{n, j} k\left(\Delta_{+} F_{j-1 / 2}^{n}\right) \varphi_{j}^{n} \\
\leq & \sum_{n, j} r h^{2} / 2 \eta^{\prime}\left(u_{j}^{n+1}\right)\left(a_{j,-}^{n} \delta_{j}^{n}+b_{j+1,+}^{n} \delta_{j+1}^{n}-a_{j-1,-}^{n} \delta_{j-1}^{n}-b_{j,+}^{n} \delta_{j}^{n}\right) \\
& +\sum_{n, j} k h r \eta^{\prime}\left(u_{j}^{n+1}\right) q\left(u_{j}^{n+1}\right) \varphi_{j}^{n} .
\end{aligned}
$$

Using (5.11), we have

$$
\left|\delta_{j}^{n}\right| \leq C h^{\alpha-1} .
$$

Taking account of (5.16), we prove that (5.15) gives as $h$ tends to 0

$$
\int_{\mathbb{R}} \int_{] 0, T[}\left[\eta(u) \varphi_{t}+F(u) \varphi_{x}\right] d x d t \geq-\int_{\mathbb{R}} \int_{] 0, T[} \eta^{\prime}(u) q(u) \varphi(x, t) d x d t
$$

that is $u$ is the entropy solution of (1.1)-(1.2). The uniqueness of this solution implies that the whole sequence $\left(u_{h}\right)$ converges towards the entropy solution $u$, of the Cauchy problem (1.1)-(1.2).

The fully second-order accurate (in space and time) may be achieved as it was proposed in [24], by advancing the cell boundary values, to be used in the flux function, to the intermediate level $t_{n+1 / 2}=t_{n}+1 / 2 k$. Using (5.4) we get the advanced bondary values :

$$
\left\{\begin{array}{l}
u_{j+1 / 2,-}^{n+1 / 2}=u_{j+1 / 2,-}^{n}-r / 2\left[f\left(u_{j+1 / 2,-}^{n}\right)-f\left(u_{j-1 / 2,+}^{n}\right)\right]+k / 2 q\left(u_{j+1 / 2,-}^{n+1 / 2}\right), \\
u_{j-1 / 2,+}^{n+1 / 2}=u_{j-1 / 2,+}^{n}-r / 2\left[f\left(u_{j+1 / 2,-}^{n}\right)-f\left(u_{j-1 / 2,+}^{n}\right)\right]+k / 2 q\left(u_{j-1 / 2,+}^{n+1 / 2}\right) .
\end{array}\right.
$$

The full semi-implicit scheme is:

$$
u_{j}^{n+1}=u_{j}^{n}-r\left[g\left(u_{j+1 / 2,-}^{n+1 / 2}, u_{j+1 / 2,+}^{n+1 / 2}\right)-g\left(u_{j-1 / 2,-}^{n+1 / 2}, u_{j-1 / 2,+}^{n+1 / 2}\right)\right]+k q\left(u_{j}^{n+1}\right) .
$$

\section{Second-order Runge-Kutta sPlitting scheme}

We focus in this section on the study of the convergence of a second-order accurate splitting scheme described in Jin [9].

As in the former sections, to ensure the existence of an entropy numerical flux, let $g$ be a numerical flux of a three point monotone scheme, as it was defined in section 2 .

We consider the splitting Runge-Kutta scheme:

$$
\begin{gathered}
u_{j}^{*}=u_{j}^{n}-\Delta t q\left(u_{j}^{*}\right), \\
u_{j}^{(1)}=u_{j}^{*}-r \Delta_{+} g_{j-1 / 2}^{*}, \\
u_{j}^{* *}=u_{j}^{(1)}+\Delta t q\left(u_{j}^{* *}\right)+2 \Delta t q\left(u_{j}^{*}\right), \\
u_{j}^{(2)}=u_{j}^{* *}-r \Delta_{+} g_{j-1 / 2}^{* *}, \\
u_{j}^{n+1}=1 / 2\left(u_{j}^{n}+u_{j}^{(2)}\right) .
\end{gathered}
$$


Proposition 6.1. Under the CFL condition:

$$
r \max _{w, z}\{|g(u, w)-g(v, w)|+|g(z, u)-g(z, v)|\} \leq|u-v| \forall u, v \in \mathcal{A}
$$

and

$$
\Delta t \sup _{u \in \mathcal{A}}\left|q^{\prime}(u)\right|<1 .
$$

The scheme (6.1-a)-(6.1-e) admits a unique solution $\left(u_{j}^{n+1}\right) \in L^{\infty}(\mathbb{Z})$, and there exist two positive constants $C_{1}$ and $C_{1}$ such that

i)

ii)

$$
\left\|u^{n+1}\right\|_{\infty} \leq C_{1}\left\|u^{0}\right\|_{\infty}
$$

$$
T V\left(u^{n+1}\right) \leq C_{2} T V\left(u^{0}\right)
$$

Proof. Let

$$
\alpha=\sup _{u \in \mathcal{A}}\left|q^{\prime}(u)\right|
$$

From (6.1-a), we have

Thus

$$
u_{j}^{*}+\Delta t q^{\prime}\left(\xi_{j}^{*}\right) u_{j}^{*}=u_{j}^{n} .
$$

Using (6.3), we have

$$
(1-\alpha \Delta t)\left|u_{j}^{*}\right| \leq\left|u_{j}^{n}\right| .
$$

$$
\left|u_{j}^{*}\right| \leq\left|u_{j}^{n}\right| /(1-\alpha \Delta t)
$$

From (6.1-b) and the properties of the used flux $g$, we obtain

$$
\left|u_{j}^{(1)}\right| \leq\left|u_{j}^{*}\right| .
$$

Hence

$$
\left|u_{j}^{(1)}\right| \leq\left|u_{j}^{n}\right| /(1-\alpha \Delta t) .
$$

(6.1-c) gives

$$
u_{j}^{* *}-\Delta t q^{\prime}\left(\xi_{j}^{* *}\right) u_{j}^{* *}=u_{j}^{(1)}+2 \Delta t q\left(u_{j}^{*}\right) ;
$$

$q^{\prime}$ being nonpositive, then

$$
\left|u_{j}^{* *}\right| \leq\left|u_{j}^{(1)}\right|+2 \alpha \Delta t\left|u_{j}^{*}\right|
$$

Making use of (6.4-a) and (6.4-b), we get

$$
\left|u_{j}^{* *}\right| \leq \frac{1+2 \alpha \Delta t}{1-\alpha \Delta t}\left|u_{j}^{n}\right| .
$$

Using again in (6.1-d), the properties of $g$ (under the CFL condition), we obtain

$$
\left|u_{j}^{(2)}\right| \leq\left|u_{j}^{* *}\right| \text {. }
$$

From (6.4-c), we deduce that

$$
\left|u_{j}^{(2)}\right| \leq \frac{1+2 \beta \Delta t}{1-\alpha \Delta t}\left|u_{j}^{n}\right| .
$$

Making use of (6.1-e) and (6.4-d), we get

$$
\left|u_{j}^{n+1}\right| \leq \frac{1+(\alpha / 2) \Delta t}{1-\alpha \Delta t}\left|u_{j}^{n}\right| .
$$


Then, we obtain

$$
\left|u_{j}^{n+1}\right| \leq\left[\frac{1+(\alpha / 2) \Delta t}{1-\alpha \Delta t}\right]^{n+1}\left|u_{j}^{0}\right| .
$$

Taking into account

$$
n \leq N=E[T /(\Delta t)]+1
$$

then

$$
\left[\frac{1+(\alpha / 2) \Delta t}{1-\alpha \Delta t}\right]^{n+1} \leq \exp [(3 / 2) \alpha T]
$$

Hence

$$
\left\|u^{n+1}\right\|_{\infty} \leq C_{1}\left\|u^{0}\right\|_{\infty}
$$

where

$$
C_{1}=\exp [(3 / 2) \alpha T]
$$

The assertion ii) related to the Total Variation may be proved by a similar method.

Theorem 6.1. If $u_{0} \in L^{\infty}(\mathbb{R}) \cap L^{1}(\mathbb{R}), f \in \mathcal{C}^{1}(\mathbb{R}), q \in \mathcal{C}^{1}(\mathbb{R})$, such that $q(0)=0$ and $q^{\prime} \leq 0$, then under (6.2) and (6.3) the approximate solution $u_{h}$ constructed by the scheme (6.1-a)-(6.1-e) converges in $L_{\text {loc }}^{1}(\mathbb{R} \times] 0, T[)$ towards the entropy satisfying solution of (1.1)-(1.2), as $h$ tends to zero.

Proof. From Proposition 6.1, the sequence $\left(u_{h}\right)$ is bounded in $L^{\infty}(\mathbb{R} \times] 0, T[) \cap$ $B V(\mathbb{R} \times] 0, T[)$; then by Helly's theorem, we can extract a subsequence still labelled $\left(u_{h}\right)$ which converges towards $u$ in $L_{l o c}^{1}(\mathbb{R} \times] 0, T[)$. Let us now prove that $u$ satisfies the entropy condition (2.3).

Let $\eta$ be any convex entropy function associated with (1.1), with entropy flux $F(u)$.

By application of the mean value theorem to (6.1-a), (6.1-c) and using the existence of an entropy numerical flux $F_{j-1 / 2}$ since (6.1-b), (6.1-d) are monotone, the equalities (6.1-a)-(6.1-e) give us

$$
\begin{gathered}
\eta\left(u_{j}^{*}\right)=\eta\left(u_{j}^{n}\right)-\Delta t q\left(u_{j}^{*}\right) \dot{\eta}\left(y_{1}\right), \\
\eta\left(u_{j}^{(1)}\right) \leq \eta\left(u_{j}^{*}\right)-r \Delta F_{j-1 / 2}^{*}, \\
\eta\left(u_{j}^{* *}\right)=\eta\left(u_{j}^{(1)}\right)+\Delta t\left[q\left(u_{j}^{* *}\right)+2 q\left(u_{j}^{*}\right)\right] \dot{\eta}\left(y_{2}\right), \\
\eta\left(u_{j}^{(2)}\right) \leq \eta\left(u_{j}^{* *}\right)-r \Delta F_{j-1 / 2}^{* *}, \\
\eta\left(u_{j}^{n+1}\right) \leq 1 / 2\left[\eta\left(u_{j}^{n}\right)+\eta\left(u_{j}^{(2)}\right)\right] .
\end{gathered}
$$

By adding (6.6-a)-(6.6-d) and multiplying by $1 / 2$, we get

$$
\begin{aligned}
\eta\left(u_{j}^{(2)}\right) & \leq \eta\left(u_{j}^{n}\right)-r / 2 \Delta_{+} F_{j-1 / 2}^{*}-r / 2 \Delta_{+} F_{j-1 / 2}^{* *} \\
& -1 / 2 \Delta t q\left(u_{j}^{*}\right) \dot{\eta}\left(y_{1}\right)+1 / 2 \Delta t\left[q\left(u_{j}^{* *}\right)+2 q\left(u_{j}^{*}\right)\right] \dot{\eta}\left(y_{2}\right) .
\end{aligned}
$$

From (6.6-e) and (6.7), we have

$$
\begin{aligned}
& \eta\left(u_{j}^{n+1}\right)-\eta\left(u_{j}^{n}\right)+r \Delta_{+}\left(1 / 2 F_{j-1 / 2}^{*}+1 / 2 F_{j-1 / 2}^{* *}\right) \\
& \quad \leq-1 / 2 \Delta t q\left(u_{j}^{*}\right) \dot{\eta}\left(y_{1}\right)+1 / 2 \Delta t\left[q\left(u_{j}^{* *}\right)+2 q\left(u_{j}^{*}\right)\right] \dot{\eta}\left(y_{2}\right) .
\end{aligned}
$$


Using a similar method as in the previous section, we get at the limit (as $h$ tends to zero) that $u$ satisfies the entropy condition (2.3) and then the whole sequence $\left(u_{h}\right)$ converges to the entropy solution of (1.1)-(1.2).

Remark 6.1. The condition (6.3) is a consequence of the presence of the negative coeffecient $(-1)$ in the first equation (6.1-a). (6.3) may be automatically satisfied if $\Delta t$ is small enough.

\section{EXTENSION TO THE TWO-Dimensional CASE}

We consider now the two-dimensional Cauchy problem:

$$
\begin{gathered}
\left.u_{t}+f_{1}(u)_{x}+f_{2}(u)_{y}=q(u) ;(x, y, t) \in \mathbb{R}^{2} \times\right] 0, T[; T>0, \\
u(x, y, 0)=u_{0}(x, y) ;(x, y) \in \mathbb{R}^{2} .
\end{gathered}
$$

In this case, we assume that

$$
u_{0} \in L^{\infty}\left(\mathbb{R}^{2}\right) \cap L^{1}\left(\mathbb{R}^{2}\right) \cap B V\left(\mathbb{R}^{2}\right) .
$$

Let $\eta(u)=|u-c|, c \in \mathbb{R}$, and

$$
\begin{aligned}
& F_{1}(u)=\operatorname{sgn}(u-c)\left(f_{1}(u)-f_{1}(c)\right), \\
& F_{2}(u)=\operatorname{sgn}(u-c)\left(f_{2}(u)-f_{2}(c)\right) .
\end{aligned}
$$

The unique entropy solution of (7.1)-(7.2) may be characterized by the following Kružkov ([11]) entropy condition:

$$
\begin{aligned}
& \int_{\mathbb{R}^{2}} \int_{] 0, T[}\left[\eta(u) \varphi_{t}+F_{1}(u) \varphi_{x}+F_{2}(u) \varphi_{y}\right] d x d y d t \\
& \geq-\int_{\mathbb{R}^{2}} \int_{] 0, T[} \operatorname{sgn}(u-c) q(u) \varphi(x, y, t) d x d y d t
\end{aligned}
$$

for all test positive functions $\varphi \in \mathcal{C}^{1}\left(\mathbb{R}^{2} \times\right] 0, T[)$, with compact support in $\left.\mathbb{R}^{2} \times\right] 0, T$.

Let

and

$$
r=\frac{\Delta t}{\Delta x}
$$

$$
s=\frac{\Delta t}{\Delta y} .
$$

We consider the numerical fluxes $g_{i}(u, v), i=1,2$, which are assumed to be locally Lipschitz functions in both arguments and such that:

1) $g_{i}(u, v)$ is nonincreasing in $u$,

2) $g_{i}(u, v)$ is nondecreasing in $v$,

3) $g_{i}(u, u)=f_{i}(u)$, for $u, v, w, z \in \mathcal{A}$ where

$$
\begin{gathered}
\mathcal{A}=\left\{v \in L^{\infty}\left(\mathbb{R}^{2}\right),\|v\|-L^{\infty}\left(\mathbb{R}^{2}\right) \leq\left\|u_{0}\right\|_{L^{\infty}\left(\mathbb{R}^{2}\right)}\right\}, \\
K_{i}^{w, z}(u, v)=\left|g_{i}(u, w)-g_{i}(v, w)\right|+\left|g_{i}(z, u)-g_{i}(z, v)\right|
\end{gathered}
$$

for $i=1,2$.

The CFL condition is

$$
r K_{1}^{w_{1}, z_{1}}(u, v)+s K_{2}^{w_{2}, z_{2}}(u, v) \leq|u-v| .
$$


For the approximation of equation (7.1)-(7.2), we often use the semi-implicit scheme:

$$
\begin{aligned}
u_{i, j}^{n+1}= & u_{i, j}^{n}-r\left[g_{1}\left(u_{i, j}^{n}, u_{i+1, j}^{n}\right)-g_{1}\left(u_{i-1, j}^{n}, u_{i, j}^{n}\right)\right] \\
& -s\left[g_{2}\left(u_{i, j}^{n}, u_{i, j+1}^{n}\right)-g_{2}\left(u_{i, j-1}^{n}, u_{i, j}^{n}\right)\right]+\Delta t q\left(u_{i, j}^{n+1}\right) .
\end{aligned}
$$

As in Propositon 3.1, we can prove

Proposition 7.1. Under the CFL condition (7.4), we have the following properties:

i) The scheme $(7.5)$ admits a unique solution $\left(u^{n+1}\right) \in L^{\infty}(\mathbb{Z} \times \mathbb{Z})$,

ii) The scheme (7.5) is monotone,

iii) $\left\|u^{n+1}\right\|_{L^{\infty}\left(\mathbb{Z}^{2}\right)} \leq\left\|u^{n}\right\|_{L^{\infty}\left(\mathbb{Z}^{2}\right)}$,

iv) $T V\left(u^{n+1}\right) \leq T V\left(u^{n}\right)$, where

$$
T V\left(u^{n}\right)=\sum_{i, j \in \mathbb{Z}}\left|u_{i+1, j}^{n}-u_{i, j}^{n}\right| \Delta y+\sum_{i, j \in \mathbb{Z}}\left|u_{i, j+1}^{n}-u_{i, j}^{n}\right| \Delta x .
$$

Theorem 7.1. If $u_{0} \in L^{\infty}\left(\mathbb{R}^{2}\right) \cap L^{1}\left(\mathbb{R}^{2}\right) \cap B V\left(\mathbb{R}^{2}\right), f_{i} \in \mathcal{C}^{1}(\mathbb{R}), q \in \mathcal{C}^{1}(\mathbb{R})$, such that $q(0)=0$ and $q^{\prime} \leq 0$, then under the CFL conditon $(7.4)$, the approximate solution $u_{h}$ constructed by the semi-implicit scheme $(7.5)$ converges in $L_{\text {loc }}^{1}\left(\mathbb{R}^{2} \times\right] 0, T[)$ towards the entropy satisfying solution of (7.1)-(7.2), as $h$ tends to zero.

Proof. The proof of this theorem is similar to that of Theorem 3.1, where we can use a result of Crandall-Majda [6] related to the existence of an entropy numerical flux in the case of the two-dimensional monotone case.

The implicit case may be analysed like the one-dimensional case in section 4 .

Remark 7.1. We did not consider here the second-order accuracy, since we know (see [8]) that we cannot construct a TVD second-order accurate scheme in the two-dimensional case.

\section{ACKNOWLEDGEMENT}

The author wishes to thank Professor J. Audounet for many interesting discussions. The author would also like to thank the referees for useful suggestions.

\section{REFERENCES}

[1] F. Benkhaldoun and A. Chalabi, Characteristic based scheme for hyperbolic conservation laws with source terms, Submitted.

[2] A. C. Berkenbosch, E. F. Kaasschieter, and J. H. M. Ten Thije Boonkkamp, The numerical wave speed for one-dimensional scalar conservation laws with source terms. Preprint, Dept. of Math. and Comp. Sci. Eindhoven University of Technology, (1994).

[3] A. Chalabi, Stable upwind schemes for hyperbolic conservation laws with source, terms, IMA J. Numer. Anal., 12 (1992), pp. 217-242. MR 93c:65108

[4] G. Q. Chen, C. D. Levermore and T. P. Liu, Hyperbolic conservation laws with stiff relaxation terms and entropy, Comm. Pure Appl. Math., 47, (1994) pp 787-830. MR 95h:35133

[5] P. Colella, A. Majda and V. Roytburd, Theoretical and numerical structure for reacting shock waves, SIAM J. Sc. Stat. Comput., 7 (1986), pp. 1059-1080. MR 87i:76037

[6] M. G. Crandall, A. Majda, Monotone difference approximations for scalar conservation conservation laws, Math. Comp. , 34, (1980), pp. 1-21. MR 81b:65079

[7] B. Engquist and B. Sjogreen, Robust difference approximations of stiff inviscid detonation waves, SIAM J. Sci. Comput., to appear. 
[8] J. B. Goodman and R. Leveque, On the accuracy of stable schemes for $2 D$ scalar conservation laws, Math. Comp., 45, (1985), pp. 15-21. MR 86f:65149

[9] S. Jin, Runge-Kutta methods for hyperbolic conservation laws with stiff relaxation terms, J. Comput. Phys., 122, (1995), pp. 51-67. MR 96g:65084

[10] S. Jin and C. D. Levermore, Numerical schemes for hyperbolic systems with stiff relaxation terms, J. Comput. Phys., Preprint.

[11] S. N. Kružkov, First order quasi-linear equations in several independent variables, Math. USSR-Sb., 10, (1970), pp. 217-243. MR 42:2159

[12] J. Leveque and H. C. Yee, A study of numerical methods for hyperbolic conservation laws with stiff source terms, J. Comput. Phys., 86, (1990), pp. 187-210. MR 90k:76009

[13] T. P. Liu, Hyperbolic conservation laws with relaxation, Commun. Math. Phys., 108, (1987), pp. 153-175. MR 88f:35092

[14] A. Majda, A qualitative model for dynamic combustion, SIAM J. Appl. Math., 40, (1981), pp.70-93. MR 82j:35096

[15] S. Osher, Riemann solvers, the entropy condition, and difference approximations, SIAM J. Numer. Anal., 21, (1984), pp.217-235. MR 86d:65119

[16] R. B. Pemper, Numerical methods for hyperbolic conservation with stiff II., SIAM J. Sci. Comput., 14, (1993), pp. 824-859.

[17] R. Sanders, On convergence of monotone finite difference schemes with variable spatial differencing., Math. of Comp., 40, (1983), pp. 91-106. MR 84a:65075

[18] H. J. Schroll, A. Tveito and R. Winther, An error bound for finite difference schemes applied to a stiff system of conservation laws, Preprint 1994-3, Dept of Informatics, University of Oslo.

[19] H. J. Schroll and R. Winther, Finite difference schemes for conservation laws with source terms, IMA J. Numer. Anal., 16, (1996), pp. 201-215. CMP 96:10

[20] C. W. Shu and S. Osher, Efficient implementation of essentially non oscillatory shock capturing schemes, J. Comput. Phys. 77, (1988), pp. 439-471. MR 89g:65113

[21] E. Tadmor, Numerical viscosity and the entropy condition for conservative difference schemes, Math. Comp. 43 (1984), pp.369-382. MR 86g:65163

[22] T. Tang and Z. H. Teng, Error bounds for fractional step methods for conservation laws with source terms, SIAM J. Numer. Anal., 32,(1995), pp. 110-127. MR 95m:65155

[23] B. Van Leer, Towards the ultimate conservative difference schemes $V$. A second order sequal to Godunov's method, J. Comput. Phys., 32, (1979), pp. 101-136.

[24] B. Van Leer, On the relation between the upwind-differencing schemes of Godunov, EngquistOsher and Roe, SIAM J. Sci. Stat. Comput., 5, (1984), pp.1-19. MR 86a:65085

[25] J. P. Vila, Convergence and error estimates in finite volume schemes for multidimensional scalar conservation laws; II Implicit monotone schemes, Preprint (1995).

[26] G. B. Whitham, Linear and nonlinear waves, J. Wiley (1974). MR 58:3905

CNRS-UMR MiP 5640 - UFR Mig Universite P. Sabatier, 118, route de Narbonne 31062 Toulouse Cedex France

E-mail address: chalabi@mip.ups-tlse.fr 